\title{
Wannier Orbital Overlap Population (WOOP), Wannier Orbital Position Population (WOPP) and the Origin of Anomalous Dynamical Charges
}

\author{
Joydeep Bhattacharjee and Umesh V Waghmare \\ Theoretical Sciences Unit \\ Jawaharlal Nehru Centre for Advanced Scientific Research \\ Jakkur PO, Bangalore 560 064, India
}

(Dated: November 1, 2007)

\begin{abstract}
Most $d^{0}$ transition metal (TM) oxides exhibit anomalously large Born dynamical charges associated with off-centering or motion of atoms along the TM-O chains. To understand their chemical origin, we introduce "Wannier Orbital Overlap Population" (WOOP) and "Wannier ond Orbital Position Population" (WOPP) based on the Wannier function based description of electronic structure obtained within first-principles density functional theory. We apply these concepts in a precise analysis of anomalous dynamical charges in $\mathrm{PbTiO}_{3}, \mathrm{BaTiO}_{3}$ and $\mathrm{BaZrO}_{3}$ in the cubic perovskite structure. Determining contributions of different atomic orbitals to the dynamical charge and their break-up into local polarizability, charge transfer and covalency, we find that $p$ orbitals of oxygen perpendicular to the -TM-O- chain contribute most prominently to the anomalous charge, by facilitating a transfer of tiny electronic charge through one unit cell from one TM atom to the next. Our results explain why the corner-shared linkage of $\mathrm{TMO}_{6}$ octahedra, as in the perovskite structure, is ideal for large dynamical charges and hence for ferroelectricity.
\end{abstract}

\section{INTRODUCTION}

Transition metal oxides (TMOs) exhibit a rich variety of structural, electric and magnetic properties 1.2 , owing mostly to the $d$ electronic states of the transition metal. Partially occupied $d$ states of a TM in its oxide result in different possibilities of magnetic ordering and properties, where as the TMOs with unoccupied $d$ states usually are non-magnetic insulators with structural and dielectric properties of technological importance ${ }^{3}$. Most ferroelectric $\left(\mathrm{ABO}_{3}\right)$ oxides in the perovskite structure ${ }^{4}$ have a $d^{0}$ transition metal at the $B$-site, and interaction between $d$ states of TM with $p$ states of $\mathrm{O}$ are known to be crucial to their properties ${ }^{5}$. Many binary $d^{0} \mathrm{TM}$ oxides such as $\mathrm{TiO}_{2}$ and $\mathrm{ZrO}_{2}$ hold promise as high- $k$ dielectric materials $\underline{6}$ essential to the future ultra-thin semiconductor devices. Understanding how precisely the $d^{0}$ state of a TM atom and its interaction with oxygen gives rise to these properties is fundamentally interesting and will also help in design of better materials.

Dielectric properties are intimately related to electric polarization (dipole moment per unit volume) of insulators. It is spontaneously present in a ferroelectric material $\underline{\underline{4}}$ even in the absence of external fields, and its coupling with structure, electric and elastic fields are central to technologically important properties of these materials. Dynamical charges, known as Born effective charges(BEC), yield polarization induced due to small displacements of atoms from their equilibrium positions. Anomalous charge is evaluated as the difference between the dynamical charge associated with an atom and the nominal charge represented by it's oxidation state. They are also the coupling constants between atomic displacements and electric field, and are relevant to the properties of materials in two important ways: (a) they often are anomalously large and indicators ${ }^{7.8}$ of the ferroelectric structural instabilities or low frequency (soft) polar phonon modes and (b) static dielectric (piezoelectric) response depends quadratically (linearly) on the effective charges and is dominated by the softest phonons indicated by the anomalous effective charges ${ }^{3}$.

First-principles calculations based on density functional theory (DFT) have been extensively used in determining dielectric ${ }^{\underline{3}}$ and ferroelectric $\underline{\underline{9}}$ properties of the $d^{0}$ transition metal oxides. Within firstprinciples DFT framework, BECs are commonly calculated using two methods: (a) Berry phase based expression of polarization and (b) DFT-linear response. Both approaches are based in the $k$-space and do not readily yield a picture in real-space of the flow of electronic charge in terms of bonding. Band-by-band decomposition $\underline{10}$ of effective charges using the linear response method was used to estimate contributions of different atomic orbitals to their anomalously large values, highlighting the role of hybridization between $d$ states of TM with $p$ states of oxygen. The band-by-band decomposition 11 of BECs associated with the $\mathrm{Ba}, \mathrm{Ti}$ and $\mathrm{O}$ atoms in $\mathrm{BaTiO}_{3}$, in groups of bands corresponding to different maximally localized Wannier functions $\frac{16}{}$, was found to be in good agreement with that obtained using the linear response based approach by Ghosez etal ${ }^{10}$. Recently, analysis based on Wannier functions ${ }^{12,13}$ has indicated the important role played by the oxygen orbitals in giving rise to large anomalous $Z^{\star}$ of the TM atoms in TMOs. However, a quantitative estimation of various contributions such as covalency, local polarizability and charge transfer to $Z^{\star}$ is still lacking.

In this work, we link Wannier function based description with atomic orbitals to quantify various chemical contributions to $Z^{\star}$. Polarization along $x$-direction is given by the trace of PxP, where $x$ 


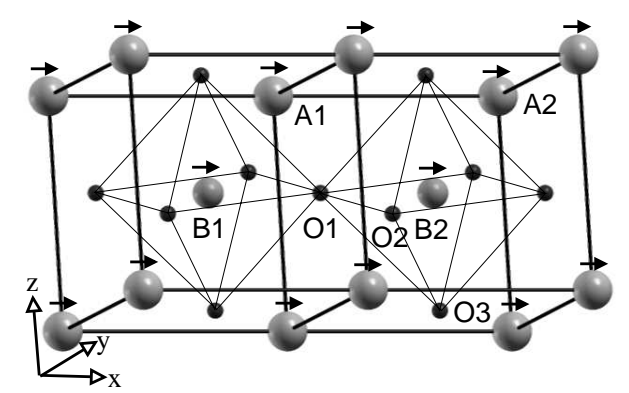

FIG. 1: Two unit cells of a cubic $\mathrm{ABO}_{3}$ perovskite system. Labels in this figure are used in discussion.

is the position operator and $P$ an operator of projection into subspace of occupied states. A natural microscopic picture of its makeup can be obtained by diagonalization of the $P x P$ operator. For systems periodic in 1-dimension, eigenfunctions of $P x P$ operator are the Wannier functions $\frac{14}{}$ (or hermaphrodite orbitals of 3 -D periodic systems $\frac{15}{15}$ ). For 3 -dimensional periodic systems, $P x P, P y P$ and $P z P$ do not generally commute and can be diagonalized simultaneously only approximately, resulting in maximally localized Wannier functions 16 . In analogy with crystal orbital overlap population $\frac{17}{1}$, based on projections of Wannier functions onto atomic orbitals, we propose here concepts of Wannier Orbital Overlap Population (WOOP) and Wannier Orbital Position Population (WOPP), which are used to determine quantitatively the contributions of different electronic mechanisms to anomalous dynamical charges. In principle, Mulliken 18 charge population becomes a subset of WOOP, if the Wannier functions are replaced by an atomic orbital basis set in which the electronic wave functions are expanded. On the other hand, WOPP introduces a new scheme to accurately estimate the degree of covalency.

In section [I] we describe the systems analyzed in this work. We review in section III] the definition of BEC, a method to construct localized Wannier Functions (WFs), and introduce WOOP and WOPP. In Section IV] we present results of our analysis and break-up of the effective charge into different mechanisms, and finally conclude in section $\mathrm{V}$.

\section{SYSTEMS}

$\mathrm{PbTiO}_{3}$ (PTO) is an end member $(x=1)$ of $\mathrm{PbZr}_{1-x} \mathrm{Ti}_{x} \mathrm{O}_{3}(\mathrm{PZT})$, the most commonly used technological ferroelectric material. It occurs in the perovskite structure with a tetragonal ferroelectric phase up to a relatively high Curie temperature $T_{c}=763 \mathrm{~K}$, above which it transforms to the cubic paraelectric phase. While the stereochemical activity of lone pair of $6 s$ electrons in $\mathrm{Pb}$ is known to be crucial for its properties, it shares with other perovskite ferroelectrics the $d^{0}$ TM-oxygen interaction contributing to ferroelectricity. It has been studied extensively using first-principles calculations $\frac{19}{}$. As indicators of ferroelectric instability, effective charges of $\mathrm{Pb}$ and $\mathrm{Ti}$ in its cubic paraelectric phase $(a=3.97 \AA)$ are 3.9 and 7.1 respectively, which are analyzed here.

$\mathrm{BaTiO}_{3}$ (BTO), a related material, undergoes a sequence of structural transitions from cubic to tetragonal, then to orthorhombic and subsequently to rhombohedral phase as temperature is reduced. In the cubic structure $(a=4.00 \AA)$, BECs of $\mathrm{Ba}$ and $\mathrm{Ti}$ are 2.74 and 7.2 respectively. Polarization of $\mathrm{BaTiO}_{3}$ is relatively small partly because Ba does not have any lone-pair of electrons and does not contribute much to ferroelectricity. A comparative study of $\mathrm{PbTiO}_{3}$ and $\mathrm{BaTiO}_{3}$ allows us to identify the special role of $\mathrm{Pb}$ at $A$-site.

Finally, we analyze effective charges in $\mathrm{BaZrO}_{3}(\mathrm{BZO})$, which remains stable in the paraelectric phase at all $\mathrm{T}$, with the cubic perovskite structure $(a=4.19 \AA)$. Effective charges of $\mathrm{Ba}$ and $\mathrm{Zr}$ are 2.74 and 5.7 respectively. Comparison of various contributions to anomalous charges of $\mathrm{Zr}$ and $\mathrm{Ti}$ allows us to single out the chemical mechanism which leads to ferroelectricity in $\mathrm{BaTiO}_{3}$, but not in $\mathrm{BaZrO}_{3}$.

\section{METHODS}

All calculations reported here have been carried out with ABINIT ${ }^{20}$ implementation of DFT using Teter extended norm-conserving pseudopotentials and a plane wave basis. The $s$ and $p$ semi-core states of $\mathrm{Ti}, \mathrm{Zr}$ and $\mathrm{Ba}$ are included in the valence. An energy cutoff of $80 \mathrm{Ry}$ on the plane-wave basis is used in representing Kohn-Sham wave functions. Integrals over the Brillouin zone have been sampled with a $6 \times 6 \times 6$ uniform mesh of k-points. Wannier functions have been constructed using post-processing subroutines interfaced with ABINIT code, developed by the authors $\underline{14}$. 


\section{A. Born effective charge}

Elements of the BECs of an atom (I) are tensors, whose elements $Z_{\alpha \beta, I}^{\star}$ are the coupling constants between atomic displacements (phonon) and electric field. Force $F$ felt by an atom $I$ in the presence of electric field $E$ is:

$$
F_{\alpha, I}=Z_{\alpha \beta, I}^{*} E_{\beta},
$$

where as the dipole moment induced by displacement $u$ of an atom is:

$$
\Delta \mu_{\alpha}=Z_{\alpha \beta, I}^{*} u_{I \beta} .
$$

Corresponding change in the polarization is $\Delta \mu / V, V$ being the volume of unit cell. In this work, we use the relation (2) in analyzing the BEC.

A well localized WF $W_{n}(\mathbf{r})$ (constructed in the scheme described below) enables evaluation of expectation values of position operator:

$$
<x>_{n}=\int x W_{n}^{\star}(\mathbf{r}, 0) W_{n}(\mathbf{r}, 0) d \mathbf{r} .
$$

The total electronic contribution to the dipole moment per unit cell is obtained by summing over all the bands: $\mu_{x}=e f \sum_{n}\langle x\rangle_{n}$, e being the electronic charge and $f$ the occupation number of the $n$th band. The BEC can be determined from the changes in the dipole moment resulting from the off-centering of an atom. For example, BEC associated with dipole induced along $x$ direction by a small displacement $u_{x}$ of an ion $I$ is given by:

$$
Z_{x x I}^{\star}=Z_{I}^{i o n}+\frac{\Delta \mu_{x}^{e}}{u_{x}}
$$

where $Z_{I}^{\text {ion }}$ is the ionic charge $\left(Z_{I}^{\text {ion }}=\right.$ number of valence electrons of the given atom treated with a pseudopotential). $\Delta \mu_{x}^{e}$ is calculated from the WCs as:

$$
\Delta \mu_{x}^{e}=e\left[\sum_{i}^{N}<x>_{i}^{d}-\sum_{j}^{N}<x>_{j}^{n d}\right],
$$

where $\langle x\rangle_{i}^{d}$ and $\left\langle x>_{i}^{n d}\right.$ are the expectation values of $x$ obtained using the $i$-th WF with and without the atomic displacement respectively. WFs thus allow decomposition of the dynamical charge into contributions from different bonding orbitals spanning the occupied subspace.

\section{B. Wannier functions}

Wannier functions(WF) are obtained as Fourier transform of Bloch functions of occupied bands. At any wave vector $\mathbf{k}$, any unitary transformation of Bloch functions within the occupied band subspace is a valid description of electronic structure in the sense that the total energy is invariant under such a transformation. As a result, WFs are highly non-unique. Nevertheless, there are always some special choices of these unitary rotations, also known as gauge, which yield highly localized sets of WFs 16,21 .

In this work, we use the Wannier type localized orbitals (WLO) described in $\operatorname{Ref}^{21}$ :

$$
W_{I}(\mathbf{r}, \mathbf{R})=\sum_{\mathbf{k}} e^{i \mathbf{k} \cdot(\mathbf{r}-\mathbf{R})} \sum_{l} M_{I l}^{\mathbf{k}} u_{l \mathbf{k}}(\mathbf{r}),
$$

where the wave functions $\left\{u_{l \mathbf{k}}(\mathbf{r})\right\}$, obtained from a DFT calculation, are the cell periodic part of Bloch functions. Rotation of these functions by the unitary matrix $M^{\mathbf{k}}$ (at each $\mathbf{k}$ ) is aimed at obtaining a set of wave functions that are smooth and periodic in reciprocal space, a criterion necessary to obtain well localized WFs.

In ionic systems, WLOs are typically centered on atoms, and their delocalized features extending to nearest neighbour atoms indicate the degree of covalency. In strongly covalent systems, WLOs are typically bond-centered, while the atom-centered WLOs can be obtained by expanding the subspace of electronic states to include the unoccupied, antibonding states. As demonstrated in Fig-2 the O1-centered [see Fig-1] WLO with $2 p_{y}$ orbital character and the Ti-centered WLO with $3 d_{x y}$ orbital character, are constructed using an extended subspace above the Fermi level, in cubic PTO. The width or "fatness" 22 of 
(a)

(b)

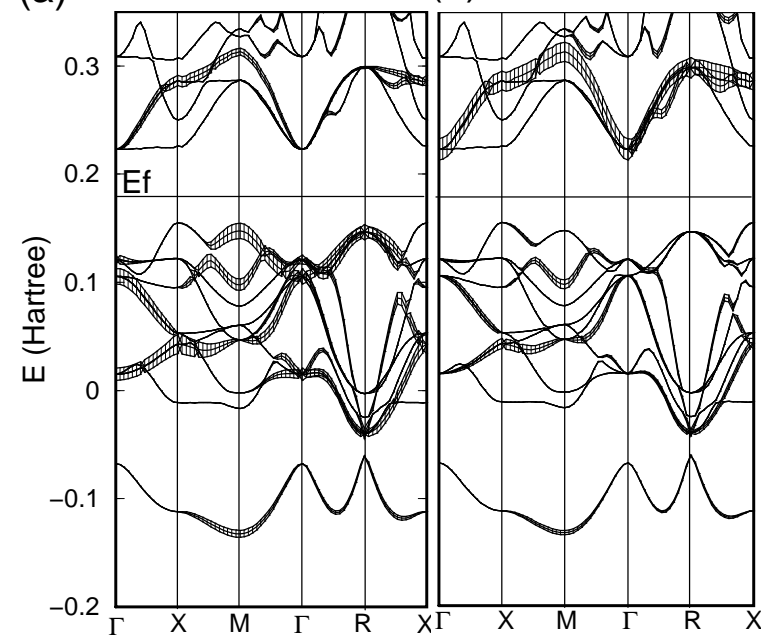

(c)

(d)

(e)

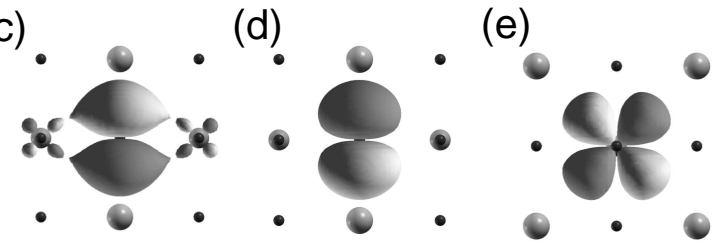

FIG. 2: Band structure of $\mathrm{PbTiO}_{3}$ with (a) $2 p_{y}$ and (b) $3 d x y$ characters centered at $\mathrm{O} 1$ and Ti respectively. The $2 p_{y}$ WLO at O1, constructed (c) within the occupied subspace, (d) in an extended subspace including bands above Ef. (e) The $3 d_{x y}$ WLO at Ti constructed in the extended subspace.

the bands in Fig-2(a) and (b) is linearly proportional to their contribution to the two WLOs respectively while Fig-2(c) shows the $2 p_{y}$ orbital of $\mathrm{O} 1$ constructed within the occupied subspace. When constructed in the extended subspace, the antibonding contribution between the orbitals $2 p_{y}$ of $\mathrm{O} 1$ and the $3 d_{x y}$ of Ti from the bands above Fermi level in Fig-2(a) destructively interfere with the corresponding bonding contributions from the bands below Fermi level to yield a pure $2 p_{y}$ like WLO shown in Fig-2(d). Whereas, for the pure Ti $3 d_{x y}$, shown in Fig-2(e), the majority contribution comes from the band above Fermi level, as clearly indicated in Fig-2(b).

In the rest of this paper, we refer to the atom-centered WLOs constructed in the extended subspace as atomic orbitals (AO) and WLOs constructed within the occupied subspace as WFs.

\section{Wannier Orbital Overlap Population (WOOP) and Wannier Orbital Position Population (WOPP)}

A picture of bonding in a crystal obtained with Wannier functions is qualitative. To link it quantitatively to different chemical mechanisms of bonding, and we now introduce tools based on their projections on atomic orbitals. For clarity, we denote indices of WFs and atomic orbitals (AO's) in upper and lower case letters respectively.

In the first step, we express the WFs in terms of AOs $\left\{\left|\phi_{j}\right\rangle\right\}$ as: $\left|W_{I}\right\rangle=\sum_{j=1}^{N} C_{I j}\left|\phi_{j}\right\rangle$, where the AOs, $N$ in number, may not necessarily form an orthonormal basis. $C_{I j}$ 's can be determined by solving $N$ simultaneous linear equations of the form:

$$
\sum_{j=1}^{N} S_{m j} C_{I j}=\left\langle\phi_{m} \mid W_{I}\right\rangle,
$$

where $S_{m j}=\left\langle\phi_{m} \mid \phi_{j}\right\rangle$. Normalization of $\left|W_{I}\right\rangle$ in terms of AOs is: $\left\langle W_{I} \mid W_{I}\right\rangle=\sum_{l m}^{N} C_{I l}^{*} S_{l m} C_{I m}$. Evidently, a choice of AOs is quite important in obtaining the normalization of WFs accurately.

The part of the summand corresponding to $l=m$ in the normalization of $\left|W_{I}\right\rangle$ gives the population of electrons at $\left|\phi_{l}\right\rangle$ due to $\left|W_{I}\right\rangle$. We generalize this to define "Wannier orbital overlap population"(WOOP) associated with the $I$-th WF as:

$$
B_{l m}^{I}=C_{I l}^{*} S_{l m} C_{I m}
$$


Choosing $l$ and $m$ corresponding to all the AOs centered on an atom, say A1 in Fig-3 the population of electrons, which are centered on $\mathrm{A} 1$ and contribute to the $W_{1}$, can be evaluated as:

$$
Q_{A 1}^{1}=\sum_{l m \in A 1} B_{l m}^{1}
$$

Subsequently, the number of electrons populated in atom A1 can be estimated as $\sum_{I} Q_{A 1}^{I}$.

Center of $\left|W_{I}\right\rangle$ can now be expressed in terms of the AOs: $\langle x\rangle_{I}=\sum_{l m} C_{I l}^{*} X_{l m} C_{I m}$, where $X_{l m}=$ $\left\langle\phi_{l}|x| \phi_{m}\right\rangle$. We refer to the summand as "Wannier orbital position population"(WOPP) and denote it as:

$$
D_{l m}^{I}=C_{I l}^{*} X_{l m} C_{I m} .
$$

This leads to the net contribution to $Z^{\star}$ from $\left|W_{I}\right\rangle$ as:

$$
Z_{x}^{\star I}=\frac{e}{u_{x}} \sum_{l, m=1}^{N}\left(D_{l m}^{d I}-D_{l m}^{n d I}\right) .
$$

Through appropriate choices of $l$ and $m$ in eqn-(11), the $Z_{x}^{\star I}$ can be easily decomposed into contributions based on different chemical mechanisms responsible. These are classified into five broad categories: rigid shift (RS), charge transfer $(\mathrm{CT}) \stackrel{10}{ }$, local polarizability (LP), covalency (COV) and other nonlocal changes (NC). For the WF centered on A1 (see schematic in Fig-3), these are defined by the following conditions on $l$ and $m$ :

$$
\begin{aligned}
& \text { 1. RS: } l=m,\{l m\} \in \mathrm{Ai}, \Delta Q_{A i}^{1}=0, i=1,2,3 ; \\
& \text { 2. CT: } l=m,\{l m\} \in \mathrm{Ai}, \Delta Q_{A i}^{1} \neq 0, i=1,2,3 ; \\
& \text { 3. LP: } l \neq m,\{l m\} \in \mathrm{A} ; \\
& \text { 4. COV: } l \neq m, l \in \mathrm{A}, m \notin \mathrm{A} \text { or } m \in \mathrm{A}, l \notin \mathrm{A} ; \\
& \text { 5. NC: } l \neq m,\{l m\} \notin \mathrm{A} .
\end{aligned}
$$

RS and CT are contributed from the diagonal blocks in Fig. 3 (identical AOs) and can be differentiated based on the change in the population $(\Delta Q)$, obtained through WOOP. Whereas, $Z^{\star}$ from the LP arises solely from the coupling between the AOs with different parities centered on the same atom, for which the inter orbital transition dipole moment is nonzero. Due to orthonormality of AOs centered on the same atom, contribution of LP to $Z^{\star}$ is not effected by the rigid translation of AOs.

Contributions to $Z^{\star}$ from COV and NC are both due to transition dipole moments between orbitals centered on different atoms. In Fig-3 where $|w 1\rangle$ has highest projection with AOs of A1, COV gives covalent contribution to $Z^{\star}$ due to transition dipole moments between the AOs of A1 and it's nearest neighbours $\mathrm{A} 2$ and $\mathrm{A} 3$. COV contributions can be both bonding as well as antibonding type in nature. Finite COV as well as CT contributions to BEC, involving same set of AOs of two neighbouring atoms, can be indicative of a possible conjugation scenario, as shown in section-IV NC gives the contribution to $Z^{\star}$ from covalency associated with transition dipole moments between the AOs of A2 and A3[Fig-3. In general, $\mathrm{NC}$ contribution to $Z^{\star}$ is much smaller than the other four categories as none of the AOs involved in $\mathrm{NC}$ belong to $\mathrm{A} 1$.

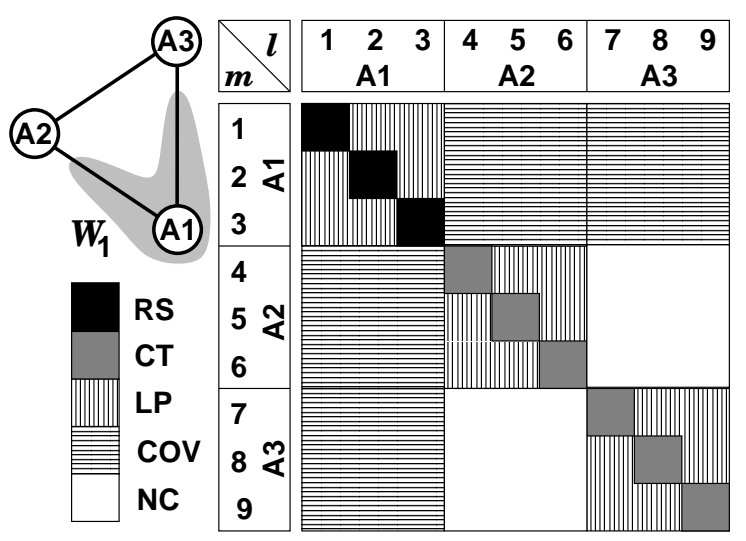

FIG. 3: A schematic grouping of $l$ and $m$ to decompose $Z^{\star}$ due to $W_{1}$ (RS: Rigid shift, CT: Charge transfer, LP: Local polarizability, COV: Covalency, NC: Nonlocal changes). 


\section{RESULTS AND DISCUSSION}

We first present WOPP based analysis of $Z_{T i}^{\star}$ in PTO and compare it with that of $Z_{T i}^{\star}$ in BTO. Next, we analyze $Z_{\mathrm{Pb}}^{\star}$ of PTO and bring out the special role played by $\mathrm{Pb}$ through comparison with $\mathrm{Ba}$. Finally, we present a comparative analysis of BTO and BZO to identify factors crucial to ferroelectricity in BTO and to the lack of it in BZO. In all these cases, cations are off-centered (with respect to the cubic structure) along $x$-axis by $1 \%$ of the lattice constant.

In addition to a nominal charge of 4 a.u., the anomalous part of $Z_{T i}^{\star}$ in $\mathrm{PbTiO}_{3}$ is about 3.1 a.u. To this anomalous part, a positive contribution of 4.2 a.u. is from the orbitals of O1, which belongs to the Ti-O chain along the $x$-axis, and a negative contribution of -1.1 a.u. from the orbitals of $\mathrm{O} 2$ and $\mathrm{O} 3$ in the Ti-O plane perpendicular to the $x$-axis. Almost $70 \%$ of the total positive contribution (4.2 a.u.) is made by the $2 p_{y}$ and $2 p_{z}$ orbitals of $\mathrm{O} 1$ (both are equal by symmetry), which are perpendicular to the Ti-O chain along $x$-axis. The O1-centered WFs with $2 p_{y}$ orbital character are shown in Fig- 4 . The majority of their contributions (see Table-I) to $Z_{T i}^{\star}$ is due to inter-atomic charge transfer across the unit cell from the $3 d_{x y}$ and $3 d_{x z}$ orbitals of Ti1 to those of Ti2. WOOP analysis for these WFs clearly shows a decrease and increase in electron population at Ti1 and Ti2 respectively, with negligible change in the population of orbitals at $\mathrm{O} 1$.

Bonding in PTO is certainly not purely ionic: a small but non-zero covalent contribution of about 0.3 a.u. to $Z^{\star}$ from mixing between the $\mathrm{O} 1$ and Ti orbitals indicates a weak $\pi$ like bonding along the Ti1-O1-Ti2 chain which, is strengthened in the ferroelectric phase. The rest $30 \%$ of the total positive contribution to the anomalous part of $Z_{T i}^{\star}$ is due to the $2 s \pm 2 p x$ hybridized orbitals of O1. Unlike the $2 p_{y}$ and $2 p_{z}$ orbitals which are perpendicular to the -O-Ti-O- chain direction, covalent contributions from these orbitals arises from $\sigma$ type bonding between $\mathrm{Ti}$ and $\mathrm{O}$ and are comparable to that from charge transfer. These WFs (shown in Fig-(5) are centered between $\mathrm{O}$ and $\mathrm{Ti}$ atoms: the $2 s+2 p_{x}$ orbital is peaked in the bond path between $\mathrm{O} 1$ and Ti1 where as $2 s-2 p_{x}$ peaked is peaked in the bond path between $\mathrm{O} 1$ and Ti2. The former contracts and the latter gets stretched in the ferroelectric phase. Consequently, in the ferroelectric phase the covalency between O1 and Ti1 gets stronger at the cost of the same between $\mathrm{O} 1$ and Ti2. This is similar to $\pi$ conjugation found in long chain conducting polymers. As shown in Table-I this results into a larger covalent type contribution to $Z_{T i}^{\star}$ from $2 s+2 p_{x}$ than that from $2 s-2 p_{x} \mathrm{WF}$. These orbitals too facilitate the transfer of small electronic charge from Ti2 to Ti1, resulting in charge transfer contributions to $Z_{T i}^{\star}$ that are about the same as their contribution with covalency. Small contributions from local polarizability of oxygen to $Z_{T i}^{\star}$ from these orbitals correlate with the intra-atomic change in hybridization among the $s, p$ and $d$ orbitals of oxygen without altering the total charge population or WOOP at O1. Similarly, local polarizability of O2 (and changes in its hybridization) yield a small negative contributions to $Z_{T i}^{\star}$.

Interestingly, charge transfer between TM atoms at $B$-site through $\mathrm{O}$ seems to be indirectly influenced by the atom A (or its size). Comparison of contributions to $Z_{T i}^{\star}$ in PTO and BTO (shown in Tables- 1 and (II) indicates that charge transfer from Ti2 to Ti1 is higher in $\mathrm{BaTiO}_{3}$ than in $\mathrm{PbTiO}_{3}$. In contrast, the contributions from the $2 s \pm 2 p_{x}$ hybridized orbitals ( $\sigma$ bonding) of $\mathrm{O} 1$ in $\mathrm{BaTiO}_{3}$ are about the same as in $\mathrm{PbTiO}_{3}$. As we will see below, the former difference is due to the fact that $\mathrm{Pb}$ also competes in contributing to the charge transfer through the same O1 orbitals.

TABLE I: Various chemical contributions to $\mathrm{Z}_{T i}^{\star}$ and $\mathrm{Z}_{P b}^{\star}$ in $\mathrm{PbTiO}_{3}$.(CP:Charge transfer; RS:Rigid shift; LP:Local polarizability; COV:Covalency; NC:Non-local changes)

\begin{tabular}{cccccc}
\hline \hline WF & CT & LP & COV & NC & Tot \\
\hline \multicolumn{5}{c}{ Decomposition of $\mathrm{Z}_{T i}^{\star}$ from orbitals of O1 } \\
\hline $2 s+2 p_{x}$ & 0.21 & -0.10 & 0.41 & 0.01 & 0.53 \\
$2 s-2 p_{x}$ & 0.33 & -0.06 & 0.30 & 0.01 & 0.58 \\
$2 p_{y}, 2 p_{z}$ & 1.10 & 0.00 & 0.27 & 0.06 & 1.43 \\
\hline \multicolumn{7}{c}{ Decomposition of $\mathrm{Z}_{P b}^{\star}$} & from orbitals of $\mathrm{O} 2$ & 0.34 \\
\hline $2 p_{x}$ & 0.12 & -0.08 & 0.28 & 0.02 & 0.01 \\
$2 p_{y} \pm 2 s$ & 0.02 & -0.04 & 0.03 & -0.00 & 0.43 \\
$2 p_{z}$ & 0.38 & -0.03 & 0.07 & 0.01 & Tot \\
\hline \multicolumn{7}{c}{ Decomposition of $\mathrm{Z}_{P b}^{\star}$ from orbitals of $\mathrm{Pb}$} & -1.43 \\
\hline $6 s$ & $\mathrm{RS}$ & $\mathrm{LP}$ & $\mathrm{COV}$ & $\mathrm{NC}$ & -0.02 \\
\hline \hline
\end{tabular}


Anomalous part of $Z_{P b}^{\star}$ in $\mathrm{PbTiO}_{3}$ is about 1.9 a.u., over a nominal charge of 2.0 a.u. Almost $25 \%$ of this anomalous part is a result of local polarizability at $\mathrm{Pb}$ due to emergence of moderate $\mathrm{Pb} 6 p$ orbital character in occupied states of the ferroelectric phase, which is accompanied by a small decrease in occupancy of the $6 s$ orbital. This is evident from the nature of deformation of the Pb-centered WF of $s$ symmetry [see Fig-6(a)] in the ferroelectric phase. The rest of the anomalous part is contributed by the two oxygens $\mathrm{O} 2$ and O3 in the (100) Ti-O plane. $2 p_{x}$ and $2 p_{z}$ symmetry WFs centered on both of these oxygen atoms give positive contributions to $Z_{\mathrm{Pb}}^{\star}$. Interaction between $\mathrm{Pb}$ and $\mathrm{O} 2$ orbitals is evident in the long-range features of the $2 p_{x}$ and $2 p_{z}$ like WF centered on O2 (shown in Fig-6 (c) and (b)) in the ferroelectric phase. The contribution from $2 p_{x}$ is primarily covalent in nature involving a combination of $6 p$ orbitals of $\mathrm{Pb}$, while the same from $2 p_{z}$ is mainly due to charge transfer from similar orbitals of $\mathrm{Pb} 2$ to that of Pb1 (see Table-I).

In both $\mathrm{BaTiO}_{3}$ and $\mathrm{BaZrO}_{3}$, the anomalous part of $Z_{B a}^{\star}$ is about 0.75 a.u. The $5 d$ orbitals of $\mathrm{Ba}$, which are unoccupied in the paraelectric phase, mix with occupied states in the ferroelectric phase giving a positive contribution to $Z_{B a}^{\star}$. These orbitals are relatively more localized than the $6 \mathrm{~s}$ or $6 \mathrm{p}$ orbitals of $\mathrm{Pb}$ and hence less favourable for inter-atomic orbital interactions. Consequently, reduction in both charge transfer as well as covalent contributions involving the $2 p$ orbitals of $\mathrm{O} 2$ and $\mathrm{O} 3$ and the $5 d$ orbitals of $\mathrm{Ba}$ is expected. Similarly, the local polarizability contribution from $\mathrm{Ba}$ due to hybridization between its $5 p$ and $5 d$ orbitals in the ferroelectric phase is also weaker. These are evident from comparison between contributions to $Z_{B a}^{\star}$ (Tables-【I and 【II) and those to $Z_{P b}^{\star}$ (Table-II). Our analysis of $Z_{Z r}^{\star}$ (Table-【II) reveals two interesting trends: (a) contribution from covalency between $\mathrm{Zr}$ and $\mathrm{O}$ ( $\sigma$-type bonding) to anomalous part of $Z_{Z r}^{\star}$ is greater than that from covalency between $\mathrm{Ti}$ and $\mathrm{O}$ to $Z_{T i}^{\star}$ and (b) charge transfer is much more significant in giving anomalous $Z_{T i}^{\star}$ than in the case of $Z_{Z \text { r }}^{\star}$.

We sum up contributions from all the WFs and summarize the decomposition of anomalous $Z^{\star}$ 's of $\mathrm{A}$ and $\mathrm{B}$ cations into various chemical mechanisms (see Table-IV). Comparison between BZO and BTO clearly reveals the role of long-range charge transfer between TM cations via $p$ orbitals of oxygen (see Fig-77). This is analogous to super-exchange in magnetic oxide materials. As expected from the fact that the radius of $\mathrm{Zr}^{4+}$ is larger than that of $\mathrm{Ti}^{4+}$, contribution to $Z^{\star}$ from covalency is greater for $\mathrm{Zr}$ than for Ti. In case of $Z_{P b}^{\star}$, the net charge transfer through the orbitals of $\mathrm{O}(2,3)$ is the prime contributor, followed by the net contribution from covalency between the orbitals of $\mathrm{O}(2,3)$ and $\mathrm{Pb}$. The local polarizability of the $6 s$ orbital of $\mathrm{Pb}$ also contributes sizeably to $Z_{P b}^{\star}$, but is partly compensated by the local polarizability of oxygen. The cause for difference between $Z_{P b}^{\star}$ and $Z_{B a}^{\star}$ is most evident in charge transfer contribution, and to a lesser extent in contributions from their local polarizability and covalency with oxygen.

\section{CONCLUSION}

We presented a simple scheme to quantify different chemical bonding mechanisms through projections of Wannier functions on to atomic orbitals. This is particularly useful in separating the total BEC into various chemical contributions such as local polarizability, covalency and charge transfer. We find that anomalously large effective charge of $\mathrm{Ti}$ in $\mathrm{BaTiO}_{3}$ and $\mathrm{PbTiO}_{3}$ arises primarily from a dynamical transfer

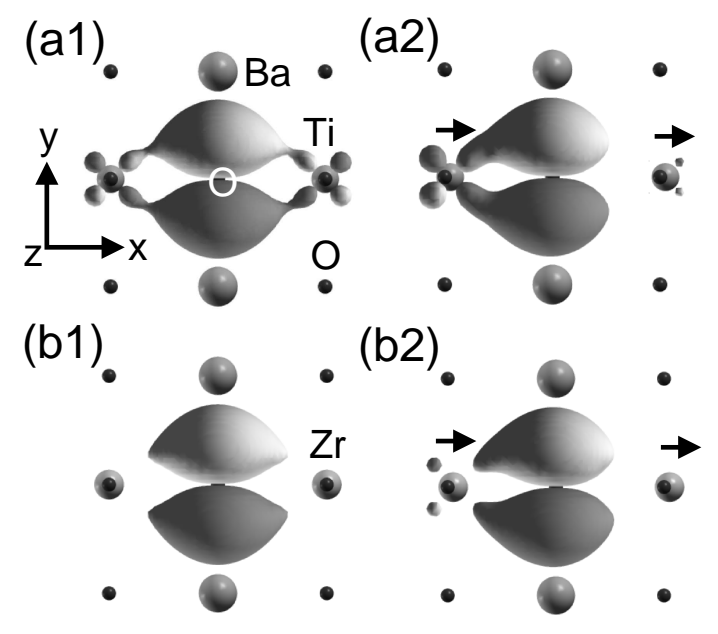

FIG. 4: O1-centered WFs with symmetry of its $2 p_{y}$ orbital in cubic (a) $\mathrm{BaTiO}_{3}$ and (b) $\mathrm{BaZrO}_{3}$ (1) with and (2) without $\mathrm{Ti} / \mathrm{Zr}$ displacement. The isosurfaces shown here, are for $|W(\mathbf{r})|=0.0045$, which is typically about $10 \%$ of its maximum. 
of tiny electronic charge through one unit cell from $d$ orbitals of one Ti atom to its nearest neighbour Ti atom in the direction opposite to Ti displacement and secondarily from covalency (the latter is less than half of the former). In contrast, the anomalous part of effective charge of $\mathrm{Pb}$ has comparable contributions from charge transfer and covalency, of which the former is lacking in the case of Ba.

We have identified the dynamical charge transfer between cations (see Fig.-77) (TM as well as the one at $A$-site) as the key factor for their potential to drive ferroelectricity. As this charge transfer occurs along -TM-O- chains through the $p$ orbitals of oxygen that are perpendicular to the chains, it is clear that perovskite is a structure very special for ferroelectricity for two reasons: (a) it has -TM-O- chains running in all the three directions and (b) its $\mathrm{TMO}_{6}$ octahedra are all corner-shared with two-fold coordination of oxygen atoms giving two of the $p$ orbitals of oxygen for facilitating this charge transfer. More generally, the tools of WOOP and WOPP developed here, will be useful in characterization of chemical bonding in variety of difference systems and phenomena.

\section{ACKNOWLEDGMENTS}

JB thanks CSIR, India for a research fellowship and UVW acknowledges a DuPont Young Faculty grant that supported some of this work. We are grateful for use of the central computing facility at JNCASR, funded by the Department of Science and Technology, Government of India.

${ }^{1}$ Rao, C. N. R.; Raveau, B. Transition Metal Oxides: Structure, Properties, and Synthesis of Ceramic Oxides, John Eiley and Sons. Inc. 1998.

2 Cox, P. A. Transition metal oxides, Clarendon Press, Oxford 1992.

${ }^{3}$ Waghmare, U. V.; Rabe, K. M. in Materials Fundamentals of Gate Dielectrics, edited by Demkov, A.; Navrotsky, A. Springer, New York 2005, 215-248.

${ }^{4}$ Lines, M. E.; Glass, A. M. Principles and Applications of Ferroelectrics and Related Materials, Clarendon, Oxford 1979.

${ }^{5}$ Cohen, R. E. Nature 1992, 258, 136-138.

6 Park, J. H.; Parise, J. B.; Woodward, P. M.; Lubomirsky, I.; Stafsudd, O. J. Mater. Res. 1999,14, $3192-3195$.

7 Zhong, W.; King-Smith, R. D.; Vanderbilt, D. Phys. Rev. Lett. 1994, 72, 3618-3621.

8 Waghmare, U. V.; Spaldin, N. A.; Kandpal, H. C.; Seshadri, R. Phys. Rev. B 2003, 67, 125111-(1-10).

9 Vanderbilt, D. Current Opinion in Solid State and Materials Science 1997, 2, 701-705.

10 Ghosez, Ph.; Michenaud, J. P.; Gonze, X. Phys. Rev. B 1998, 58, 6224-6240.

11 Marzari, N.; Vanderbilt, D. Unpublished

12 Cangiani, G.; Baldereschi, A.; Posternak, M.; Krakauer, H. Phys. Rev. B 2004, 69, 121101-(1-4).

13 Posternak, M.; Baldereschi, A.; Walter, E. J.; Krakauer, H. Phys. Rev. B 2006, 74, 125113-(1-8).

14 Bhattacharjee, J.; Waghmare, U. V. Phys. Rev. B 2005, 71, 045106-(1-5).

15 Sgiarovello, C.; Peressi, M.; Resta, R. Phys Rev B 2001, 64, 115202-(1-10).

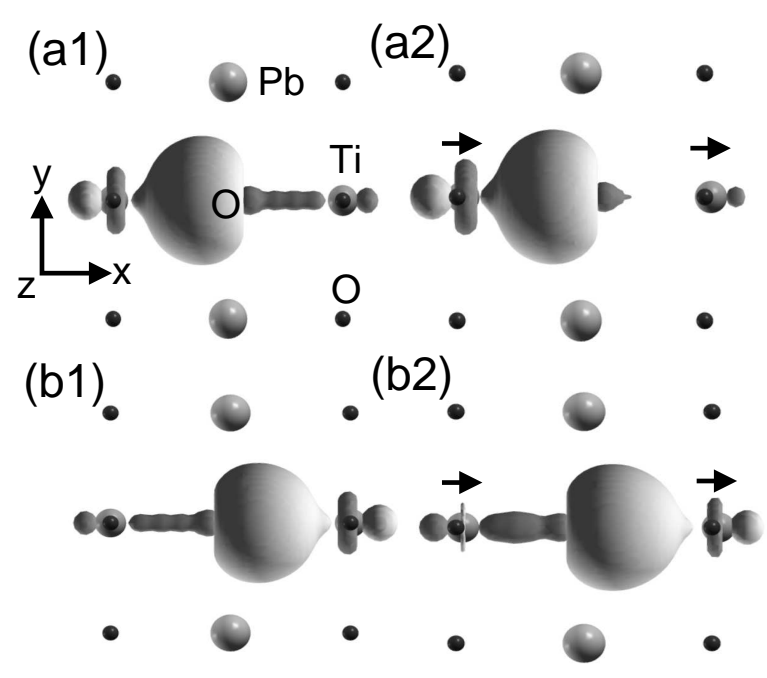

FIG. 5: O1-centered WFs with symmetries of (a) $2 s+2 p_{x}$ and (b) $2 s-2 p_{x}$ orbitals (1) with and (2) without Ti displacement in cubic $\mathrm{PbTiO}_{3}$. The isosurfaces are for $|W(\mathbf{r})|=0.0045$, which is about $10 \%$ of its maximum. 


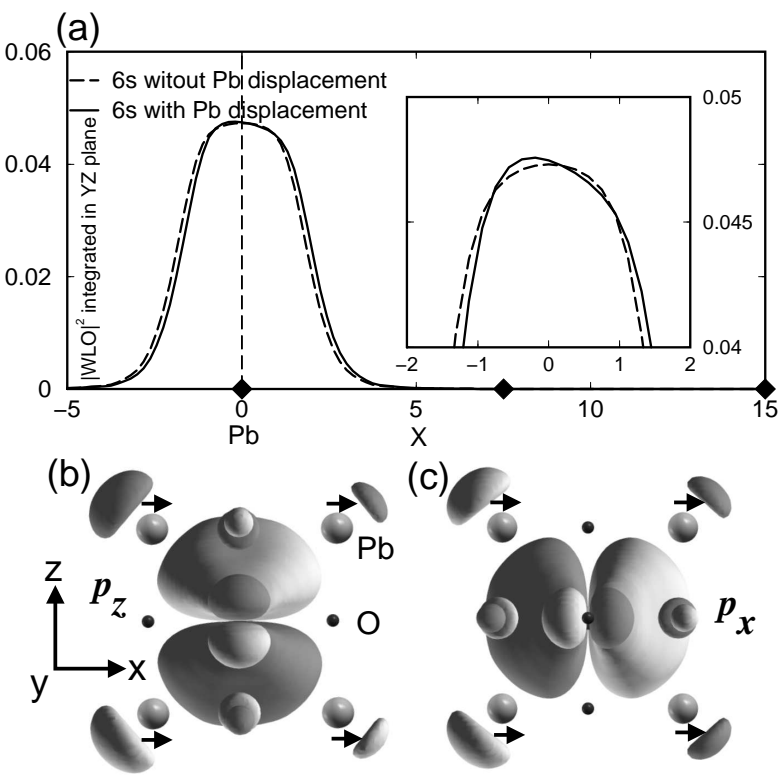

FIG. 6: WFs with symmetry of (a) $6 s$ orbital of $\mathrm{Pb}$, (b) $2 p_{z}$ and (c) $2 p_{x}$ orbitals of $\mathrm{O} 1$ in cubic $\mathrm{PbTiO}_{3}$. Isosurfaces shown in (b) and (c) are for $|W(\mathbf{r})|=0.001$.

16 Marzari, N.; Vanderbilt, D. Phys. Rev. B 1997, 56, 12847-12865 .

17 Hoffman, R. Rev. Mod. Phys. 1988 , 60, 601-628.

18 R. S. Mulliken, R. S. J. Chem. Phys.,1955, 23, 1833-1840

19 Waghmare, U. V.; Rabe, K. M. Phys. Rev. B 1996, 55, 6161-6173.

20 First-principles computation of material properties: the ABINIT software project, Gonze, X. etal, Computational Materials Science 2002, 25, 478-492.

21 Bhattacharjee, J.; Waghmare, U. V. Phys. Rev. B 2006, 73, 121102(R)-(1-4).

22 Jepsen, O.; Andersen, O. K. Z. Phys. B: Condens Matter 1995, 97, 35-47.

TABLE II: Various chemical contributions to $\mathrm{Z}_{T i}^{\star}$ and $\mathrm{Z}_{B a}^{\star}$ in $\mathrm{BaTiO}_{3}$.

\begin{tabular}{cccccc}
\hline \hline WF & CT & LP & COV & NC & Tot \\
\hline \multicolumn{7}{c}{ Decomposition of $\mathrm{Z}_{T i}^{\star}$ from orbitals of O1 } \\
\hline $2 s+2 p_{x}$ & 0.19 & -0.08 & 0.42 & -0.01 & 0.52 \\
$2 s-2 p_{x}$ & 0.34 & -0.05 & 0.29 & -0.00 & 0.58 \\
$2 p_{y}, 2 p_{z}$ & 1.18 & -0.01 & 0.26 & 0.06 & 1.49 \\
\hline \multicolumn{7}{c}{ Decomposition of $\mathrm{Z}_{B a}^{\star}$ from orbitals of $\mathrm{O} 2$} \\
\hline $2 p_{x}$ & -0.01 & -0.05 & 0.31 & 0.01 & 0.26 \\
$2 s \pm 2 p_{y}$ & 0.04 & -0.04 & 0.06 & -0.00 & 0.06 \\
$2 p_{z}$ & 0.11 & 0.00 & 0.11 & -0.00 & 0.22 \\
\hline \multicolumn{7}{c}{ Decomposition of $\mathrm{Z}_{B a}^{\star}$ from orbitals of Ba } \\
\hline $\mathrm{WF}$ & $\mathrm{RS}$ & $\mathrm{LP}$ & $\mathrm{COV}$ & $\mathrm{NC}$ & Tot \\
\hline $5 p_{x}$ & -1.94 & 0.08 & -0.02 & 0.00 & -1.88 \\
$5 p_{y}, 5 p_{z}$ & -1.94 & 0.09 & -0.00 & 0.00 & -1.85 \\
\hline \hline
\end{tabular}




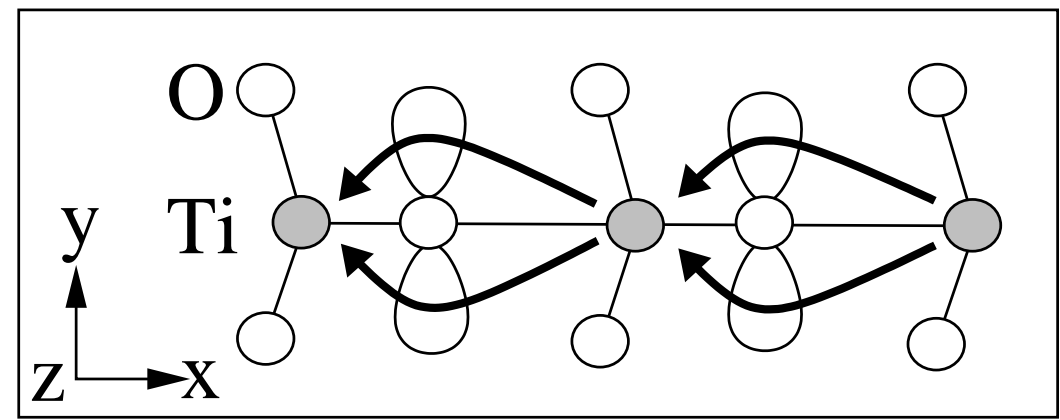

FIG. 7: A schematic picture of interatomic charge transfer across one unit cell.

TABLE IV: Comparison of various contributions to anomalous effective charges.

\begin{tabular}{lcccc}
\hline \hline & $\begin{array}{c}\text { Charge } \\
\text { transfer }\end{array}$ & Covalency & \multicolumn{2}{c}{$\begin{array}{c}\text { Local } \\
\text { polarizability }\end{array}$} \\
\hline & & (from orbitals of O1) & \\
$\mathrm{Ti}(\mathrm{BTO})$ & 2.89 & 1.23 & -0.15 & \\
$\mathrm{Zr}(\mathrm{BZO})$ & 1.61 & 1.57 & $(\mathrm{O} 2+\mathrm{O} 3)$ & -0.28 \\
\hline & & $(\mathrm{O} 2+\mathrm{O} 3+\mathrm{A})$ & -0.26 & 0.26 \\
$\mathrm{Ba}(\mathrm{BTO})$ & 0.36 & 1.06 & -0.38 & 0.58 \\
$\mathrm{~Pb}(\mathrm{PTO})$ & 1.08 & 0.80 & & \\
\hline \hline
\end{tabular}

TABLE III: Various chemical contributions to $\mathrm{Z}_{Z r}^{\star}$ and $\mathrm{Z}_{B a}^{\star}$ in $\mathrm{BaZrO}_{3}$.

\begin{tabular}{cccccc}
\hline \hline WF & CT & LP & COV & NC & Tot \\
\hline \multicolumn{7}{c}{ Decomposition of $\mathrm{Z}_{Z_{r}}^{\star}$} & from orbitals of O1 \\
\hline $2 s+2 p_{x}$ & -0.16 & -0.10 & 0.63 & 0.00 & 0.37 \\
$2 s-2 p_{x}$ & -0.01 & -0.10 & 0.50 & 0.01 & 0.40 \\
$2 p_{y}, 2 p_{z}$ & 0.89 & -0.04 & 0.22 & 0.03 & 1.10 \\
\hline \multicolumn{7}{c}{ Decomposition of $\mathrm{Z}_{B a}^{\star}$} & from orbitals of O2 \\
\hline $2 p_{x}$ & 0.03 & -0.03 & 0.23 & 0.02 & 0.25 \\
$2 s \pm 2 p_{y}$ & 0.04 & -0.03 & 0.04 & -0.00 & 0.05 \\
$2 p_{z}$ & 0.13 & 0.00 & 0.07 & -0.01 & 0.19 \\
\hline \multicolumn{7}{c}{ Decomposition of $\mathrm{Z}_{B a}^{\star}$ from orbitals of Ba } \\
\hline $\mathrm{WF}$ & $\mathrm{RS}$ & $\mathrm{LP}$ & $\mathrm{COV}$ & $\mathrm{NC}$ & Tot \\
\hline $5 p_{x}$ & -1.93 & 0.07 & -0.03 & -0.00 & -1.89 \\
$5 p_{y}, 5 p_{z}$ & -1.94 & 0.07 & -0.00 & -0.00 & -1.87 \\
\hline \hline
\end{tabular}

